\title{
Maternal embryonic leucine zipper kinase inhibitor 0TSSP167 has preclinical activity in multiple myeloma bone disease
}

\author{
Joséphine Muller, ${ }^{1, a}$ Arnold Bolomsky, ${ }^{2, a}$ Sophie Dubois, ${ }^{1}$ Elodie Duray, ${ }^{1}$ \\ Kathrin Stangelberger, ${ }^{2}$ Erwan Plougonven, ${ }^{3}$ Margaux Lejeune, ${ }^{1}$ \\ Angélique Léonard, ${ }^{3}$ Caroline Marty, ${ }^{4}$ Ute Hempel, ${ }^{5}$ Frédéric Baron, ${ }^{1,6}$ \\ Yves Beguin,, ${ }^{1,6}$ Martine Cohen-Solal, ${ }^{4}$ Heinz Ludwig, ${ }^{2}$ Roy Heusschen ${ }^{1, b}$ \\ and Jo Caers ${ }^{1,6, b}$
}

${ }^{1}$ Laboratory of Hematology, GIGA-I3, University of Liège, Belgium; ${ }^{2}$ Wilhelminen Cancer Research Institute, Department of Medicine I, Wilhelminenspital, Vienna, Austria; ${ }^{3}$ PEPs (Products, Environments, Processes), Chemical Engineering, Liège, Belgium; ${ }^{4}$ INSERMUMR-1132, Université Paris Diderot, France; Institute of Physiological Chemistry, Faculty of Medicine Carl Gustav Carus, Technische Universität Dresden, Germany and ${ }^{6}$ Department of Hematology, $\mathrm{CHU}$ de Liège, Belgium

aJoséphine Muller and Arnold Bolomsky are co-first authors. ${ }^{\circ}$ Roy Heusschen and Jo Caers are co-senior authors.
Haematologica 2018

Volume 103(8):1359-1368

\section{ABSTRACT}

M ultiple myeloma bone disease is characterized by an uncoupling of bone remodeling in the multiple myeloma microenvironment, resulting in the development of lytic bone lesions. Most myeloma patients suffer from these bone lesions, which not only cause morbidity but also negatively impact survival. The development of novel therapies, ideally with a combined anti-resorptive and bone-anabolic effect, is of great interest because lesions persist with the current standard of care, even in patients in complete remission. We have previously shown that MELK plays a central role in proliferation-associated high-risk multiple myeloma and its inhibition with OTSSP167 resulted in decreased tumor load. MELK inhibition in bone cells has not yet been explored, although some reports suggest that factors downstream of MELK stimulate osteoclast activity and inhibit osteoblast activity, which makes MELK inhibition a promising therapeutic approach. Therefore, we assessed the effect of OTSSP167 on bone cell activity and the development of myeloma-induced bone disease. OTSSP167 inhibited osteoclast activity in vitro by decreasing progenitor viability as well as via a direct anti-resorptive effect on mature osteoclasts. In addition, OTSSP167 stimulated matrix deposition and mineralization by osteoblasts in vitro. This combined anti-resorptive and osteoblast-stimulating effect of OTSSP167 resulted in the complete prevention of lytic lesions and bone loss in myeloma-bearing mice. Immunohistomorphometric analyses corroborated our in vitro findings. In conclusion, we show that OTSSP167 has a direct effect on myeloma-induced bone disease in addition to its antimultiple myeloma effect, which warrants further clinical development of MELK inhibition in multiple myeloma.

\section{Introduction}

The development of lytic bone lesions due to multiple myeloma bone disease (MMBD) is a hallmark of multiple myeloma (MM). ${ }^{1} \mathrm{MMBD}$ occurs in more than $80 \%$ of $\mathrm{MM}$ patients ${ }^{2}$ and is caused by an uncoupling of bone remodeling. $\mathrm{MMBD}$ not only results in morbidity but also directly stimulates MM tumor growth through multiple mechanisms, resulting in a vicious cycle of bone destruction and MM growth. ${ }^{3,4}$ Although novel therapies continue to increase the life expectancy for MM patients, lytic bone lesions in these patients rarely heal. ${ }^{4}$ Bisphosphonates are the current standard of care for MMBD but can be respon-

\section{Correspondence:}

jo.caers@chu.ulg.ac.be

Received: November 26, 2017.

Accepted: May 3, 2018.

Pre-published: May 10, 2018.

doi:10.3324/haematol.2017.185397

Check the online version for the most updated information on this article, online supplements, and information on authorship \& disclosures: www.haematologica.org/content/103/8/1359

\section{(C)2018 Ferrata Storti Foundation}

Material published in Haematologica is covered by copyright. All rights are reserved to the Ferrata Storti Foundation. Use of published material is allowed under the following terms and conditions:

https://creativecommons.org/licenses/by-nc/4.0/legalcode. Copies of published material are allowed for personal or internal use. Sharing published material for non-commercial purposes is subject to the following conditions:

https://creativecommons.org/licenses/by-nc/4.0/legalcode, sect. 3. Reproducing and sharing published material for commercial purposes is not allowed without permission in writing from the publisher. 
sible for side effects such as osteonecrosis of the jaw, renal impairment, atypical fractures and hypocalcemia. .,6 $^{\circ}$ These limitations highlight the need for new therapeutic strategies that ideally have a combined anti-MM and anti-MMBD effect.

We recently reported that maternal embryonic leucine zipper kinase (MELK) expression is strongly associated with proliferative high-risk MM, and that MELK inhibition with a small molecule inhibitor, OTSSP167, reduces tumor load in a murine MM model. ${ }^{7}$ Overexpression of MELK as well as an inverse correlation between MELK expression and survival has been reported for multiple malignancies. ${ }^{8-10}$ MELK promotes cell cycle progression and interacts with $\mathrm{M}$-phase inducer phosphatase 2 (CDC25B) and co-localizes with key cell cycle regulators such as cyclin B1 and cyclin-dependent kinase 1 (CDK1). ${ }^{11}$ Downstream targets of MELK include the transcription factor forkhead box protein M1 (FOXM1) 12 and the histone-methyltransferase enhancer of zeste homolog 2 (EZH2). ${ }^{13}$ Of note, FOXM1 can also directly regulate MELK expression, ${ }^{10}$ presumably resulting in a positive feedback loop, and has been identified as a therapeutic target for high-risk $M M{ }^{14}$

The role of MELK and FOXM1 in osteoclasts and osteoblasts has not yet been explored. Regarding EZH2, Fang et al. showed that EZH2 promotes osteoclastogenesis by reducing the transcription factor IRF8 and subsequent upregulation of NFATC $1 .{ }^{15}$ In addition, EZH2 prevents osteogenic differentiation of mesenchymal cells and suppression of runt-related transcription factor 2 (RUNX2) has been implicated in this process. ${ }^{16}$ Inhibition of EZH2 has been shown to mitigate bone loss in ovariectomized mice ${ }^{17}$ and to reverse $\mathrm{MM}$-induced suppression of osteoblast differentiation in vitro. ${ }^{18}$

Because the described roles of downstream targets of MELK suggest that its inhibition could concurrently block osteoclast function and stimulate osteoblast function, we examined the potential of OTSSP167 as a novel therapeutic agent for MMBD.

\section{Methods}

\section{Reagents}

OTSSP167 (Biorbyt) was dissolved in DMSO and stored at $20^{\circ} \mathrm{C}$. For in vivo studies, OTSSP167 was dissolved in $0.5 \%$ methylcellulose (Sigma-Aldrich) and stored at $-20^{\circ} \mathrm{C}$. The following antibodies were used: anti-FOXM1 (SC-502, Santa Cruz), anti-EZH2 (\#4905, Cell Signaling Technology) anti-MELK (GTX111958, GeneTex and 2274S, Cell Signaling Technology), anti- $\alpha$-tubulin (T6074, Sigma), anti-GAPDH (2118, Cell Signaling Technology), anti-rabbit-HRP (P0217, Agilent) and anti-mouse HRP (P0260, Agilent).

\section{Cells and culture conditions}

Human peripheral blood mononuclear cells (PBMCs) were obtained after Ficoll (GE Healthcare) separation of whole blood. RAW264.7 cells and 5TGM.1GFP+ cells were cultured in DMEM (Lonza) supplemented with $10 \%$ fetal bovine serum (FBS)(Sigma-Aldrich), 2mM L-glutamine (Lonza) and 1\% penicillin/streptomycin (P/S) (Lonza). TERT ${ }^{+}$bone marrow mesenchymal stromal cells (BMSC-TERT) (kindly provided by Dr. D Campana, St. Jude Children's Research Hospital, Memphis, TN, USA) were cultured in RPMI-1640 (Gibco) supplemented with $10 \%$ FCS, $2 \mathrm{mM} \mathrm{L}$-glutamine and $1 \% \mathrm{P} / \mathrm{S}$.

\section{Cell viability assay and cell cycle analysis}

RAW264.7 and PBMC viability were assessed with the cell proliferation kit I (Roche). BMSC-TERT viability was assessed with the Cell Counting Kit 8 (Sigma-Aldrich). For cell cycle analysis, cells were stained using PI/RNase staining buffer (BD Biosciences), followed by FACS analysis on a FACSCalibur (BD Biosciences).

\section{Osteoclast differentiation and in vitro bone matrix resorption}

PBMCs were seeded at a density of 750,000 cells $/ \mathrm{cm}^{2}$ in alphaMEM (Lonza) supplemented with 10\% FCS, 2 mM L-glutamine and $1 \% \mathrm{P} / \mathrm{S}$. Cells were left to adhere for 4 hours. Next, the medium was refreshed and supplemented with $25 \mathrm{ng} / \mathrm{ml}$ human $\mathrm{M}$ CSF and $50 \mathrm{ng} / \mathrm{ml}$ human sRANKL (Peprotech). The culture medium was refreshed twice per week and cultures were stopped on day 14. RAW264.7-derived osteoclast cultures were established as described previously. ${ }^{19}$ TRAP activity in osteoclast cultures was detected using the Leukocyte TRAP kit (Sigma-Aldrich). Alternatively, cultures were lysed for RNA or protein extraction. Bone resorption by osteoclasts was assessed in Osteo Assay 96well plates (Corning) as described previously. ${ }^{19}$ Actin ring formation was assessed by staining cultures with phalloidin-FITC (Sigma-Aldrich), followed by analysis on an A1R confocal fluorescent microscope (Nikon).

\section{Quantification of reactive oxygen species}

Reactive oxygen species (ROS) were detected using the Cellular Reactive Oxygen Species Detection Assay kit (Abcam). In short, cells were stained with DCDFA for 30 minutes at $37^{\circ} \mathrm{C}$ and fluorescence was measured $(\mathrm{Ex} / \mathrm{Em}=485 / 535 \mathrm{~nm})$ on an Infinite M200 Pro plate reader (Tecan).

\section{Osteoblast differentiation and functional analyses}

BMSC-TERT were seeded at a density of $25,000 \mathrm{cells} / \mathrm{cm}^{2}$ and grown to $70-80 \%$ confluence. Osteoblast differentiation was initiated by changing the medium to alpha-MEM supplemented with $10 \%$ FCS, 2 mM L-glutamine, 1\% P/S, $100 \mathrm{nM}$ dexamethasone, $50 \mu \mathrm{g} / \mathrm{ml}$ ascorbic acid and $3 \mathrm{mM} \beta$-glycerophosphate (Sigma-Aldrich). Osteogenic medium was refreshed twice per week. Collagen secretion and matrix mineralization were assessed by Sirius Red and Alizarin Red staining, respectively, as described previously. ${ }^{19,20}$

\section{Real-time PCR}

Real-time PCR was performed as described previously ${ }^{19}$ using $250 \mathrm{nmol} / \mathrm{L}$ of the appropriate primers (IDT, Online Supplementary Table S1) or pre-designed Taqman gene expression assays (Applied Biosystems). Gene expression was normalized to $\beta$-actin and $\beta 2$ microglobubulin expression (osteoclasts) or RPLPO (osteoblasts). Measurements were performed at least in triplicate and relative expression levels were determined using the $\Delta \mathrm{Ct}$ method.

\section{Western blotting}

Cells were lysed in RIPA Lysis and Extraction buffer (Thermo Scientific) supplemented with cOmplete Protease Inhibitor Cocktail (Roche). Twenty $\mu$ g of protein were separated by gel electrophoresis on a 10\% SDS-polyacrylamide gel and transferred onto PVDF membranes (BioRad). Membranes were blocked with $5 \% \mathrm{BSA} / \mathrm{PBS} /$ Tween 20 and incubated overnight at $4^{\circ} \mathrm{C}$ with primary antibodies (MELK: 1:1000, FOXM1: 1:100, EZH2: 1:1000, $\alpha$-tubulin: 1:5000, GAPDH: 1:2000). The next day, blots were incubated with a HRP-conjugated secondary antibody (1:5000), followed by visualization on an ImageQuant LAS4000 (GE Healthcare). 
Treatment of myeloma-bearing mice with OTSSP167

We used the C57BL/KaLwRij 5TGM.1 mouse mode ${ }^{21}$ to assess the effect of OTSSP167 on the development of MMBD. Nineweek-old female mice were injected i.v. with $5.0 \times 10^{5}$ 5TGM.1GFP + cells and OTSSP167 or vehicle solution was administered by oral gavage at different dose levels. For every cohort, mice were randomly divided into a naive group (no $M M, n=5$ ), a vehicle group ( $M M+$ vehicle, $n=10)$ and a treated group ( $M M+$ OTSSP167, $\mathrm{n}=10$ ). When mice showed signs of active myeloma, i.e., paraplegia, all mice within a cohort were sacrificed. Bone marrow plasmocytosis was determined by flushing the bones of one leg followed by flow cytometry on a FACSCanto flow cytometer (BD Biosciences). The femur and tibia of the opposite leg were defleshed and stored in 70\% EtOH. Ethical approval was obtained for all mouse experiments (ULg license no. 1336).

\section{Micro-computed tomography}

Micro-computed tomography $(\mu \mathrm{CT})$ was performed on distal femurs with the Skyscan 1172 system (Bruker), as described previously. ${ }^{19} 3 \mathrm{D}$ models of bones were generated using CTVol software (Bruker). The number of cortical perforations $>50 \mu \mathrm{m}$ in diameter was counted blinded on reconstructed images.

\section{Bone histomorphometry}

Femurs were embedded in methylmethacrylate. All parameters were recorded as recommended by the American Society for Bone
A
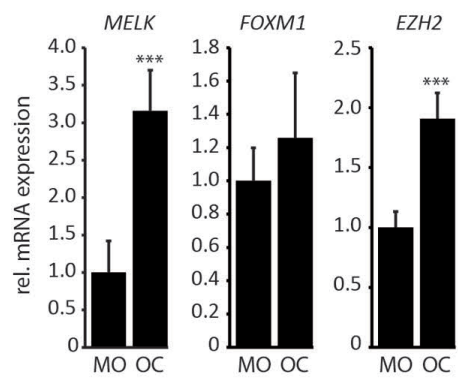

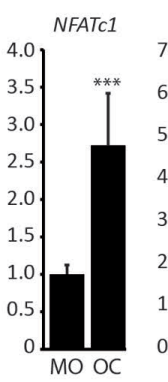

B

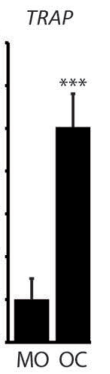

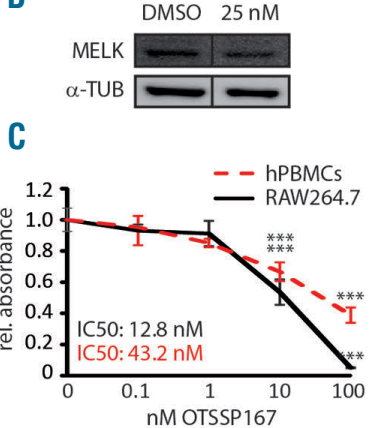

D

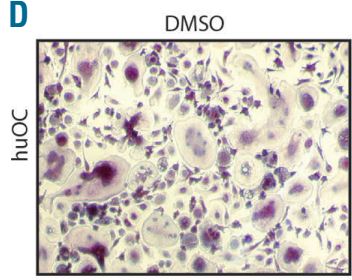

$\mathrm{E}$

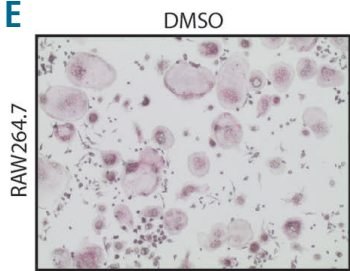

$\mathrm{F}$

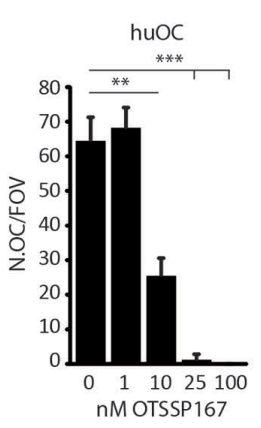

1 nM OTSSP 167

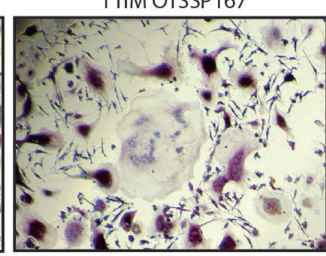

$0.1 \mathrm{nM}$ OTSSP 167

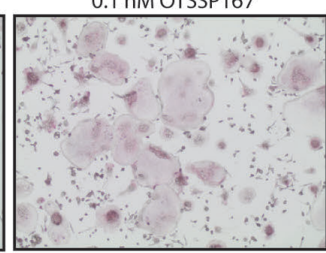

G

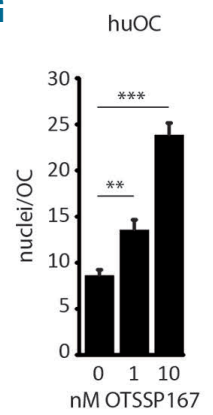

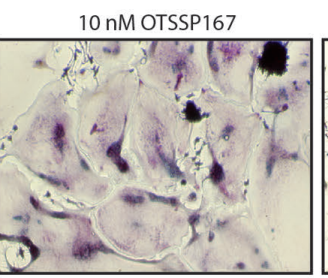

1 nM OTSSP 167

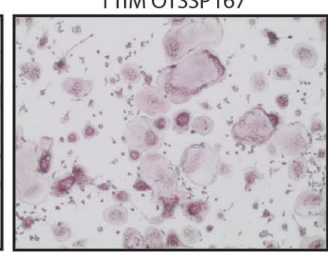

H

RAW264.7

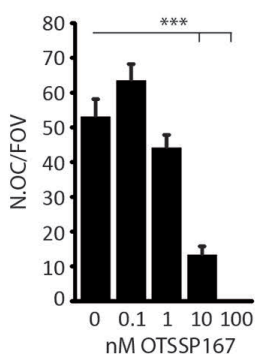

25 nM OTSSP 167

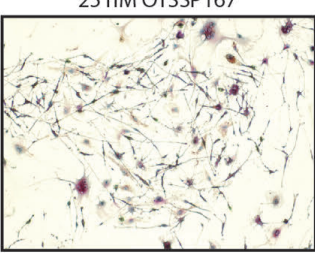

$10 \mathrm{nM}$ OTSSP 167

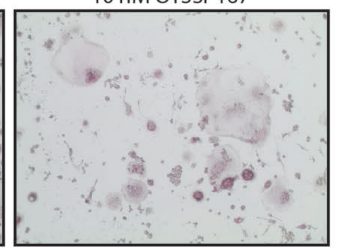

I

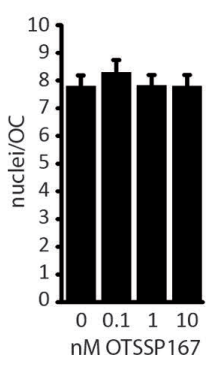

Figure 1. OTSSP167 hampers osteoclast differentiation. A) mRNA levels of MELK, EZH2, FOXM1 and relevant transcription factors during osteoclastogenesis in murine cultures, MO: monocytes; OC: osteoclasts. B) The effect of OTSSP167 treatment on MELK protein expression in RAW264.7 cultures. C) MTT assay on PBMCs and RAW264.7 cells incubated with a range of OTSSP167 concentrations. D+E) Representative images of TRAP-stained human (huOC) and RAW264.7-derived osteoclast cultures continuously treated with a range of OTSSP167 concentrations. F) Quantification of osteoclast numbers per field of view (N.OC/FOV) in huOC cultures. G) Number of nuclei per osteoclast in huOC cultures. H) Quantification of N.OC/FOV in RAW264.7-derived cultures. I) Number of nuclei per osteoclast in RAW264.7derived cultures. All data are represented as mean $+/-$ standard error. $*: P<0.05, * *: P<0.01, * * *: P<0.001$. For clarity, only significant differences with vehicletreated cultures are shown. 
A

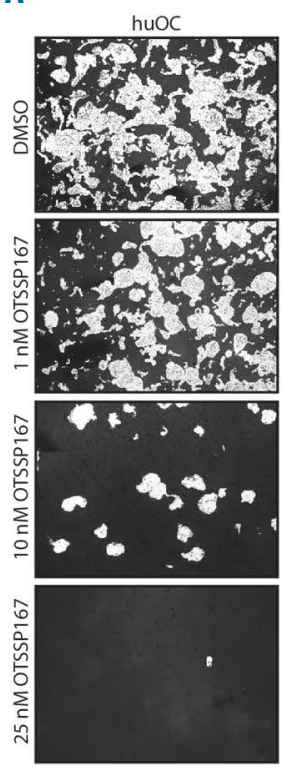

B

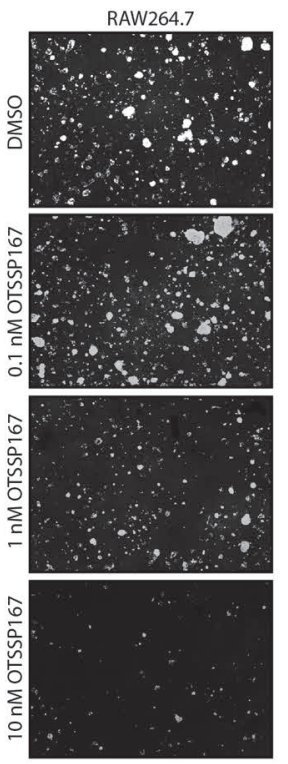

C
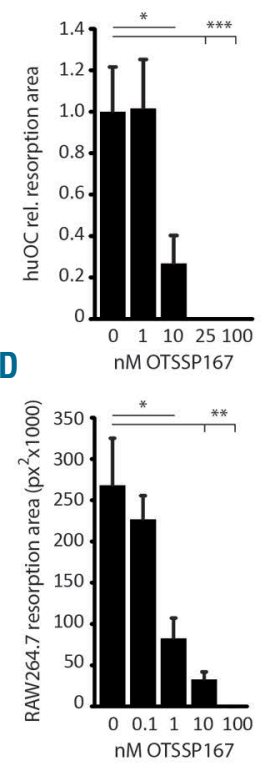

E

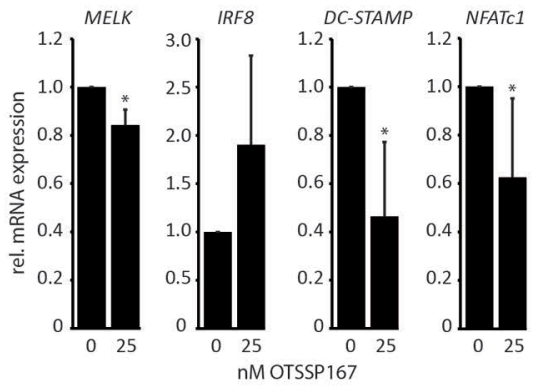

F
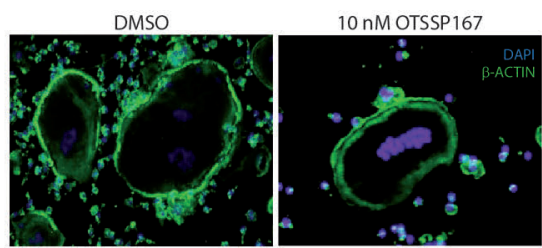

Figure 2. OTSSP167 inhibits bone matrix resorption. A+B) Representative images of Von Kossa-stained bone matrix resorption assays of huOC and RAW264.7derived osteoclast cultures continuously treated with a range of OTSSP167 concentrations. C+D) Quantification of the matrix resorption area in huOC and RAW264.7derived osteoclast cultures. E) Real-time PCR analysis of MELK, IRF8, DC-STAMP and NFATc1 following OTSSP167 treatment of RAW264.7-derived osteoclast cultures. F) Representative confocal microscopy images of phalloidin-FITC-stained RAW264.7-derived osteoclast cultures. All data are represented as mean +/- standard error. $*: P<0.05, * *: P<0.01, * * *: P<0.001$. For clarity, only significant differences with vehicle-treated cultures are shown.

and Mineral Research Histomorphometry Nomenclature Committee. ${ }^{22}$ Osteoblast surface and osteoid surface were measured on sections stained with toluidine blue and Masson's trichrome (Sigma-Aldrich). Osteoclasts were detected by TRAP staining (Sigma-Aldrich) and osteoclast surface was determined. Briefly, sections were stained for acid phosphatase using naphthol ASTR phosphate as substrate in the presence of $50 \mathrm{mM}$ tartrate with hexazotised pararosaniline, and counterstained with methyl green. Measurements were performed in NDP.View 2.6.13.

\section{Statistical analyses}

All experiments were performed at least in triplicate. Results are shown as means $+/$ - standard error and representative images are shown. For comparisons of 2 means, a Student $t$-test was used. For comparisons of multiple means, a one-way ANOVA was used, followed by a Dunnett's post-hoc test or Tukey's post-hoc test. All statistical analyses were performed with Prism 5 (Graphpad software). $P$-values below 0.05 were considered significant and $P$-values are represented as follows: ${ }^{*}=P<0.05,{ }^{* *}=P<0.01,{ }^{* * *}=P<0.001$.

\section{Results}

\section{TSSP167 hampers osteoclast differentiation}

During normal osteoclastogenesis, MELK levels increased in osteoclasts cultured from murine RAW264.7 cells (Figure 1A) or from primary human mononuclear cells (results not shown) when compared to monocyte cultures (Figure 1A). We could confirm the earlier described ${ }^{15}$ osteoclast activation pathway (EZH2-IRF8-NFATC1) by showing significant increases in EZH2, NFATC1, TRAP and CTSK and a decrease in IRF8 mRNA (Figure 1A). We subsequently studied the effects of the MELK inhibitor OTSSP167 on the proliferation of osteoclast progenitor cells and on the differentiation into osteoclasts. Treatment of RAW264.7 cells with 25 nM OTSSP167 for 24 hours resulted in decreased MELK protein levels (Figure 1B). Because of the described role of MELK in cell cycle progression, we assessed the effect of continuous OTSSP167 treatment on RAW264.7 and human PBMC viability, and found that the viability of both osteoclast progenitor populations decreased (IC50: $12.8 \mathrm{nM}$ and $43.2 \mathrm{nM}$, respectively) (Figure 1C). This coincided with an induction of G2/M cell cycle arrest (Online Supplementary Figure S1A). The decrease in progenitor viability corresponded with a decrease in both human (Figure 1D and 1F) and murine (Figure $1 \mathrm{E}$ and $1 \mathrm{H}$ ) osteoclast differentiation following continuous OTSSP167 treatment. Although the number of osteoclasts decreased, osteoclast size was markedly increased in human cultures treated with $10 \mathrm{nM}$ OTSSP167, with a corresponding increase in the number of nuclei per osteoclasts (Figure 1G). This was not the case for RAW264.7 osteoclast cultures (Figure 1I).

\section{TSSP167 inhibits bone matrix resorption}

Bone matrix resorption decreased following OTSSP167 treatment of both primary human (Figure $2 \mathrm{~A}$ and $2 \mathrm{C}$ ) and murine (Figure $2 \mathrm{~B}$ and $2 \mathrm{D}$ ) osteoclast cultures. Of note, this effect was apparent in human osteoclasts treated with 10 nM OTSSP167, suggesting that the enlarged osteoclasts that are present at this concentration are not efficiently resorbing matrix. Similarly, murine osteoclast bone resorption was inhibited at $1 \mathrm{nM}$ OTSSP167, a concentration at which no effect on osteoclast differentiation was observed. The blocking effect of OTSSP167 on osteoclast differentiation was confirmed at mRNA level, where we observed an increased expression of the negative regulator IRF8 and decreased expression of NFATC1 and DCSTAMP (Figure 2E). On the 
A

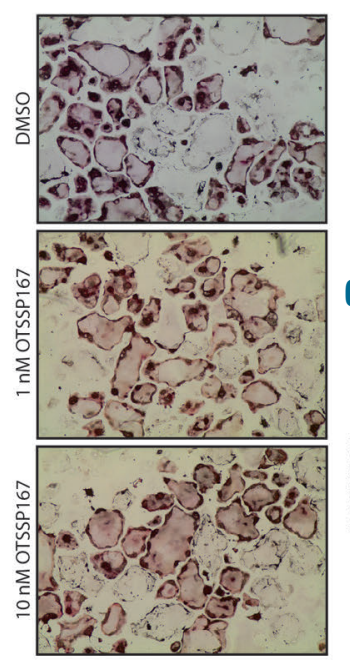

B

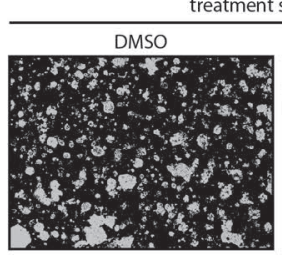

C

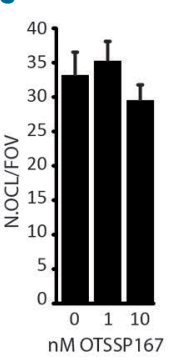

treatment start: day 5

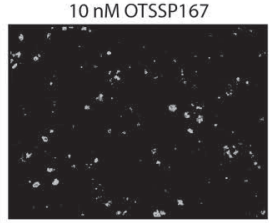

$\mathrm{E}$

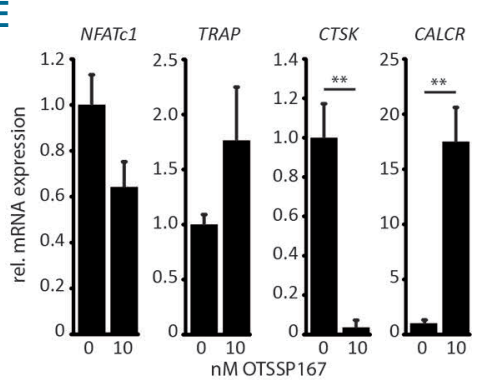

treatment start: day 7

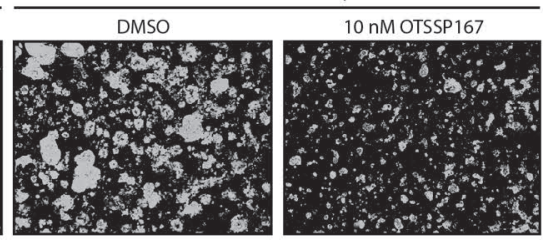

$\mathrm{F}$

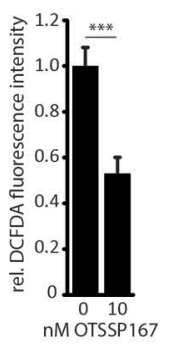

Figure 3. Established osteoclast cultures are sensitive to OTSSP167. A) Representative images of TRAP-stained RAW264.7-derived osteoclast cultures treated with a range of OTSSP167 concentrations. Treatment of these cultures was initiated after the final day of osteoclast differentiation (day 5). B) Representative images of Von Kossa-stained bone matrix resorption assays of huOC and RAW264.7-derived osteoclast cultures treated with a range of OTSSP167 concentrations. Treatment of these cultures was initiated when mature osteoclasts appeared (early: day 5, late: day 7). C) Quantification of osteoclast numbers per field of view (N.OC/FOV) in RAW264.7-derived cultures treated with OTSSP167 starting at day 5. D) Quantification of the matrix resorption area in RAW264.7-derived osteoclast cultures treated with OTSSP167 starting at day 5 or day 7. E) Real-time PCR analysis of osteoclast differentiation markers following OTSSP167 treatment starting at day 5 of RAW264.7-derived osteoclast cultures. F) ROS generation by RAW264.7-derived osteoclasts treated with OTSSP167 starting on day 5 . All data are represented as mean +/- standard error. *: $P<0.05, * *: P<0.01, * * *: P<0.001$.

other hand, we detected an increase in EZH2 mRNA and protein levels, while the phosphorylation level of FOXM1 decreased (Online Supplementary Figure S1B). Finally, we observed no difference in actin ring formation in mature osteoclasts following OTSSP167 treatment (Figure 2F).

\section{Established osteoclast cultures are sensitive to 0TSSP167}

In order to confirm that the effects of OTSSP167 on osteoclast function are not solely due to a decrease in monocyte viability, we initiated treatment of mature RAW264.7 osteoclasts after the final day of differentiation (day 5). In this setting, treatment with up to $10 \mathrm{nM}$ OTSSP167 had no effect on the number of osteoclasts (Figure $3 \mathrm{~A}$ and $3 \mathrm{C}$ ). Initiation of OTSSP167 treatment on day 5 or day 7 of in vitro matrix resorption assays markedly reduced bone matrix resorption (Figure $3 \mathrm{~B}$ and $3 \mathrm{D}$ ). Mature osteoclasts appeared at day 5 in these cultures and the presence of mature osteoclasts at the end of both DMSO- and OTSSP167-treated cultures was confirmed (not shown). Twenty-four hour treatment of mature osteoclasts had no impact on NFATc1 or TRAP mRNA levels (Figure 3E). However, we found OTSSP167 greatly reduced CTSK expression and greatly increased calcitonin receptor $(C A L C R)$ expression by mature osteoclasts (Figure 3E). Finally, as multiple reports indicate that both osteoclast differentiation and bone resorption by osteoclasts depend on the generation of reactive oxygen species (ROS), ${ }^{23,24}$ we assessed whether OTSSP167 could hamper the generation of ROS by mature osteoclasts and this was indeed the case (Figure 3F).

\section{TSSP167 stimulates osteoblast function}

Contrary to osteoclasts, MELK, EZH2 and FOXM1 expression decreased during $\mathrm{OB}$ formation indicating an inhibitory role in $\mathrm{OB}$ development (Figure 4A). These data suggest an opposing role for these molecules in $\mathrm{OB}$ formation which is in line with the results obtained with OTSSP167. Treatment of BMSC-TERT with OTSSP167 for 24 hours resulted in decreased MELK, EZH2 and FOXM1 protein levels (Figure 4B). Although monocyte viability was considerably reduced following OTSSP167 treatment in the low nanomolar range, bone marrow stromal cell viability was unaffected at these concentrations (IC50: $800.2 \mathrm{nM}$ ) (Figure 4C). Accordingly, no G2/M cell cycle arrest was detected (Online Supplementary Figure S1A). Functionally, OTSSP167 increased collagen deposition by osteoblasts (Figure 4D and 4E) and induced a marked increase in matrix mineralization (Figure 4D and 4F). Finally, we determined the effect of OTSSP167 on osteoblast marker gene expression and found that osterix (OSX) expression was increased. Conversely RUNX2, osteopontin (OPN) and interleukin-6 (IL-6) expression were decreased (Figure 4G).

\section{Preclinical activity of 0TSSP167 in multiple myeloma bone disease}

Our in vitro data indicate that OTSSP167 blocks osteoclast activity while stimulating osteoblast activity. Therefore, we assessed the potential of OTSSP167 as a novel therapy for MMBD in vivo in the 5TGM.1 MM model. The effects of OTSSP167 on myeloma development are given and discussed in detail in a separate publication. ${ }^{7}$ OTSSP167 treatment prevented the development of MMBD and this effect was similar at all dosing schedules tested (Figure 5A). OTSSP167 reduced the number of cortical perforations in MM-bearing mice (Figure 5B), without affecting cortical thickness (Ct.Th) (Online Supplementary Figure S1E). The loss of trabecular bone volume (Tb.BV/TV) that occurs in MM-bearing mice com- 
pared to healthy controls was completely prevented following treatment with OTSSP167 (Figure 5C). This was due to an increase in the number of trabeculae (Tb.N) (Figure 5D) and a decrease in trabecular separation (Tb.Sp) (Online Supplementary Figure S1F). As a result, trabecular connectivity density (Conn.Dn) was similar to healthy mice in OTSSP167-treated MM-bearing mice (Online
Supplementary Figure S1G). Trabecular thickness was not affected in MM-bearing mice (Online Supplementary Figure $S 1 H)$. Importantly, the observed prevention of MMBD in these mice does not appear to be solely due to a decreased tumor load following OTSSP167 treatment, as MMBD was prevented at a dose $(7.5 \mathrm{mg} / \mathrm{kg} / 2 \mathrm{~d})$ which had no effect on tumor load (Figure 5E).

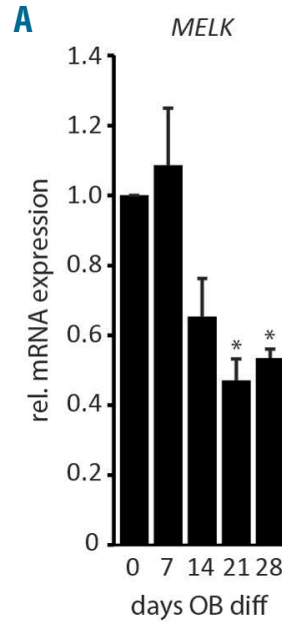

D
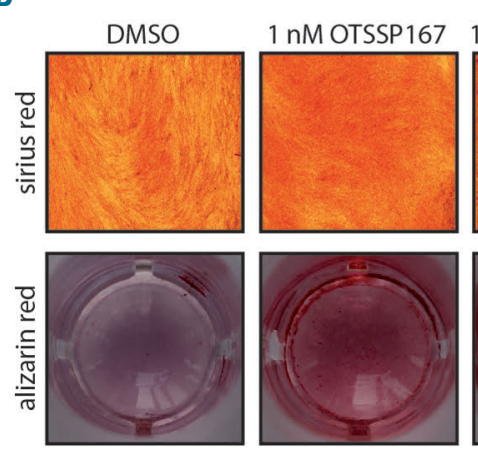

G

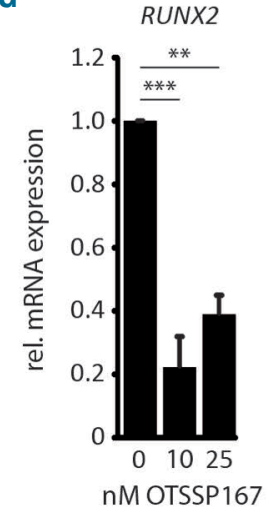

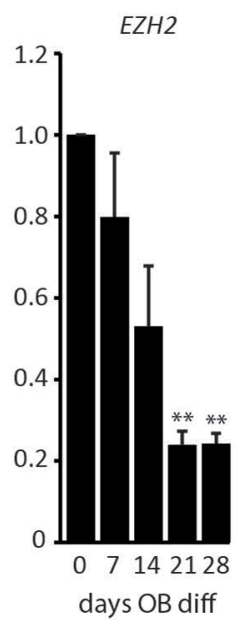

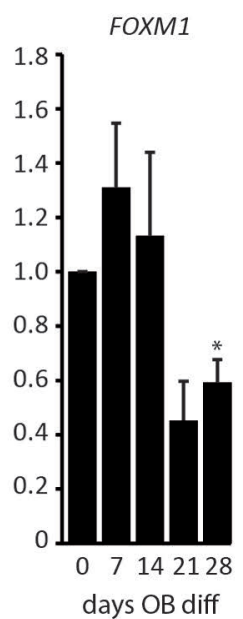

B

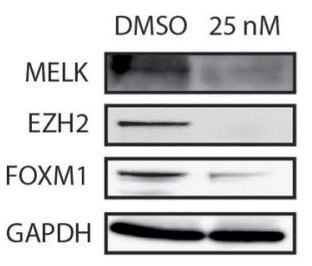

C

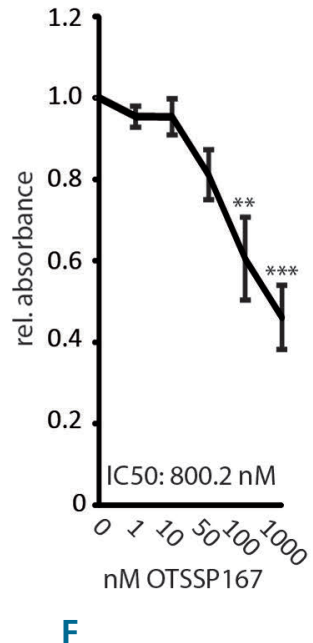

E

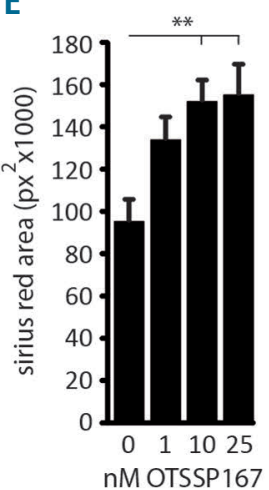

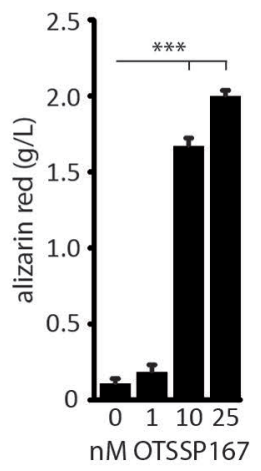
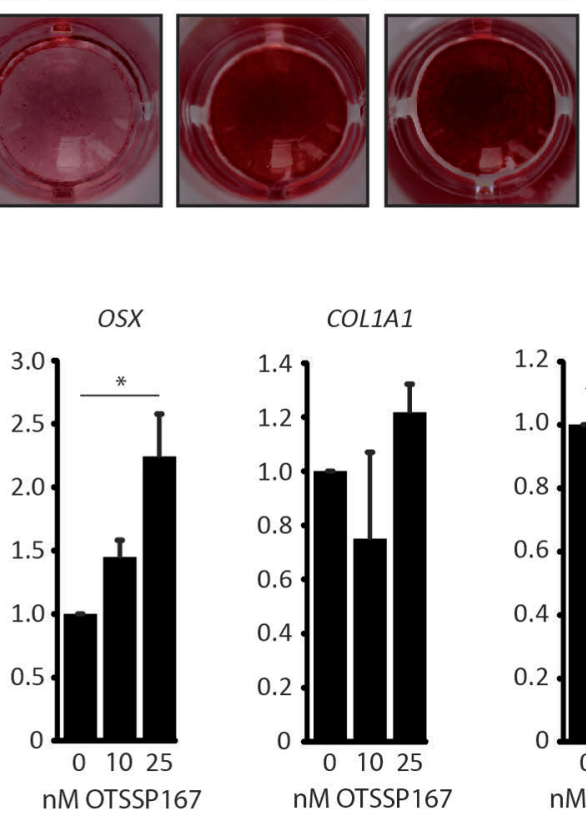

COLIA1

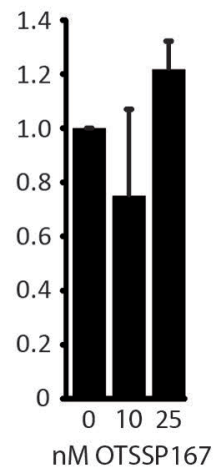

$A L P$

OPN

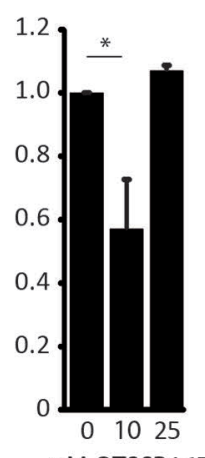

nM OTSSP 167

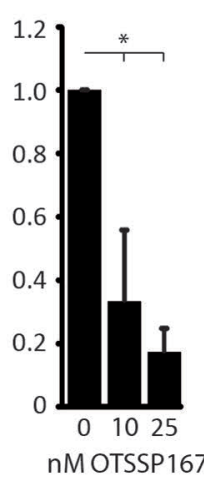

IL-6

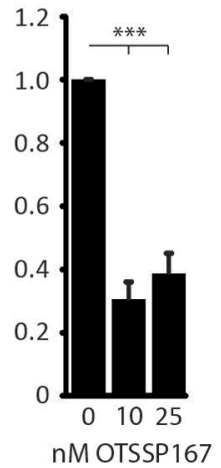

Figure 4. OTSSP167 stimulates osteoblast function. A) MELK, EZH2 and FOXM1 mRNA levels during osteoblast differentiation of BMSC-TERT cells. B) The effect of OTSSP167 treatment on MELK, EZH2 and FOXM1 protein levels in BMSC-TERT osteoblast cultures. C) MTT assay on BMSC-TERT cells incubated with a range of OTSSP167 concentrations. D) Representative images of sirius red (top panels) and alizarin red (bottom panels) stainings of BMSC-TERT osteoblast cultures treated with a range of OTSSP167 concentrations. E) Quantification of collagen deposition by BMSC-TERT osteoblasts following OTSSP167 treatment. F) Quantification of matrix mineralization by BMSC-TERT osteoblasts following OTSSP167 treatment. G) Real-time PCR analysis of osteoblast differentiation marker expression by BMSCTERT osteoblasts following OTSSP167 treatment. All data are represented as mean $+/$ - standard error. $*: P<0.05, * *: P<0.01, * * *: P<0.001$. Except for panel A, only significant differences with vehicle-treated cultures are shown for clarity. 
OTSSP167 decreases osteoclast activity and restores osteoblast activity in multiple myeloma-bearing mice

Because of the lack of an anti-myeloma effect with an OTSSP167 dose of $7.5 \mathrm{mg} / \mathrm{kg} / 2 \mathrm{~d}$, we performed immunohistomorphometry on the bones of mice from this cohort. The increase in osteoclast surface observed in MM-bearing mice was completely prevented after OTSSP167 treatment (Figure 6A and 6D). Together with an increase in osteoclast activity, osteoblast surface was suppressed in MM-bearing mice and OTSSP167 restored osteoblast surface levels to those observed in naive mice (Figure 6B and 6E). Finally, given the effect of OTSSP167 on osteoblast collagen deposition and mineralizing activity in vitro, we analyzed osteoid and found that OTSSP167 increased osteoid surface in MM-bearing mice (Figure 6C and 6F). In addition, osteoid thickness was reduced in OTSSP167-treated MM-bearing mice (Figure 6G).
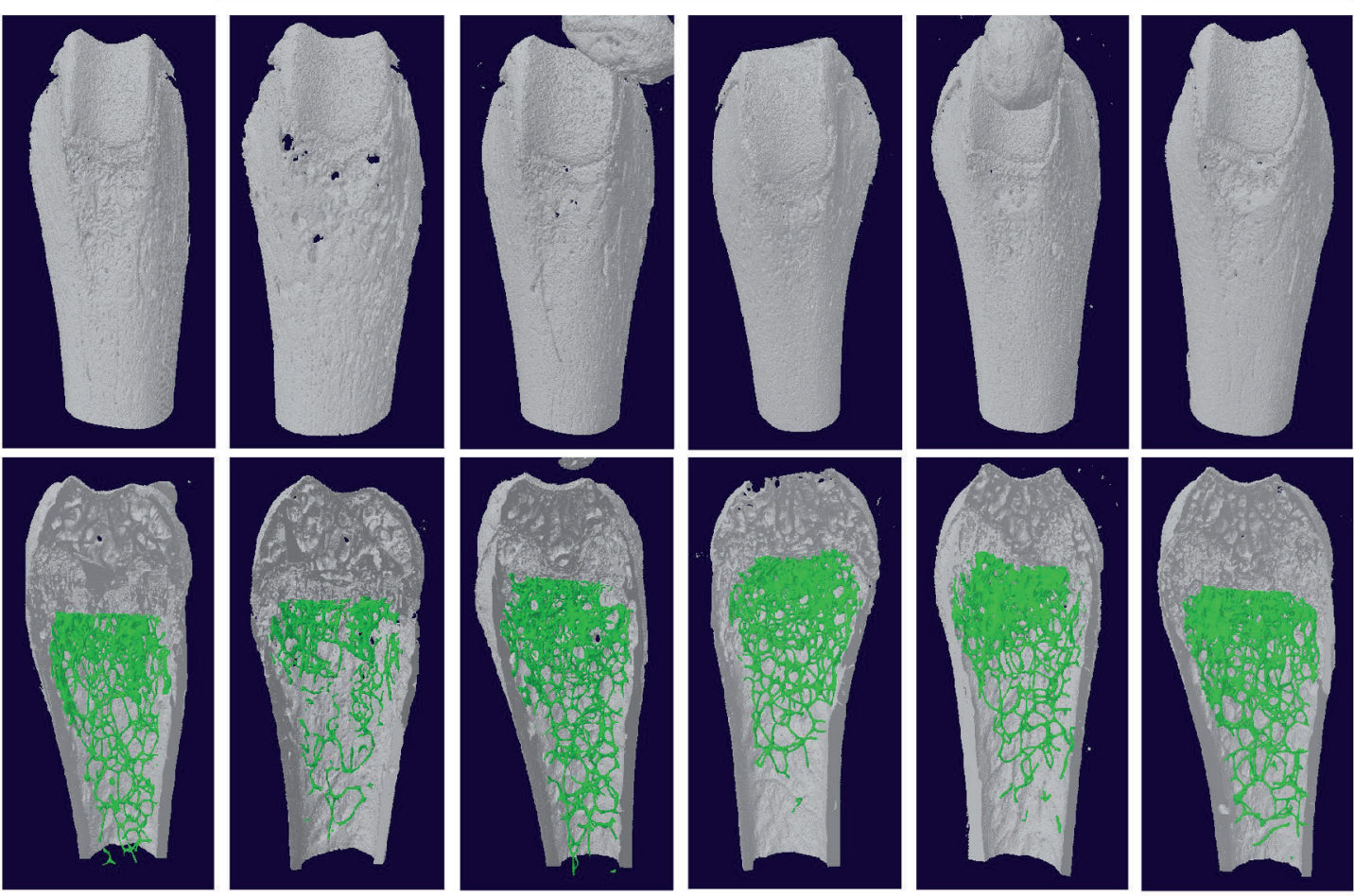

naive

B

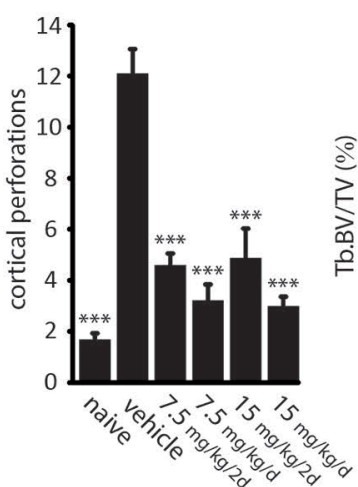

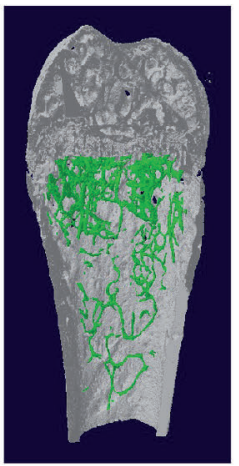

vehicle

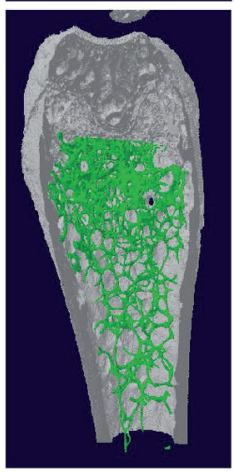

$7.5 \mathrm{mg} / \mathrm{kg} / 2 \mathrm{~d}$ OTSSP 167

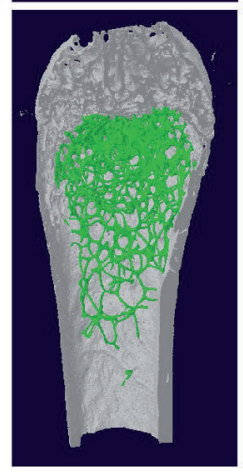

$7.5 \mathrm{mg} / \mathrm{kg} / \mathrm{d}$ OTSSP 167

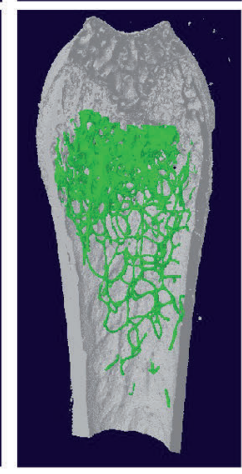

$15 \mathrm{mg} / \mathrm{kg} / 2 \mathrm{~d}$ OTSSP 167

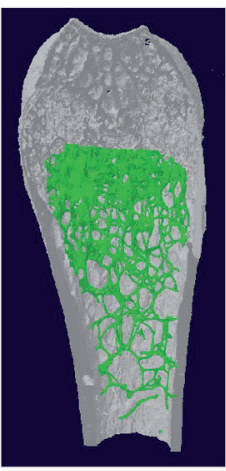

$15 \mathrm{mg} / \mathrm{kg} / \mathrm{d}$ OTSSP167

E
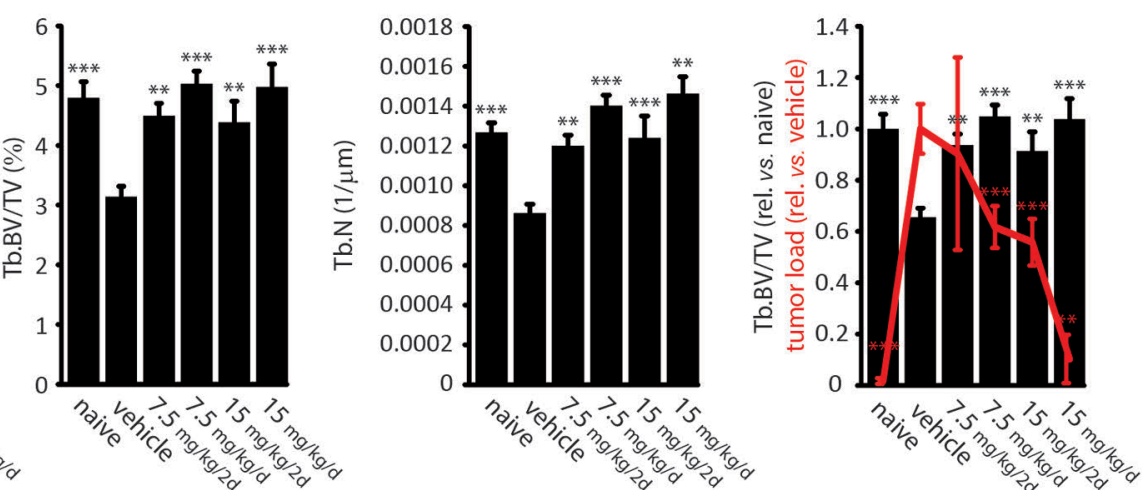

\footnotetext{
Figure 5. Preclinical activity of OTSSP167 in multiple myeloma bone disease. A) Representative 3D-reconstructions of distal femurs of naive or 5TGM.1 MM-bearing mice treated with vehicle solution or various dosing schedules of OTSSP167. Upper panels show a frontal view. Lower panels show a frontal section of the frontal view. Cortical bone is colored white, trabecular bone is colored green. B) Quantification of the number of cortical perforations with a diameter of at least $50 \mu \mathrm{m}$. CTAn analysis was performed and C) trabecular bone volume (Tb.BV/TV), D) trabecular number (Tb.N), E) Superimposition of tumor load data (red line, relative to vehicle-treated MM-bearing mice) and Tb.BV/TV (black bars, relative to naive mice) in the different mouse cohorts. All data are represented as mean $+/$ - standard error. All statistical differences are reported versus vehicle-treated mice. No difference between the different dosing schedules was observed. $*: P<0.05, * *: P<0.01$, $* * *: P<0.001$.
} 


\section{Discussion}

In this preclinical study, we demonstrated that MELK inhibitor OTSSP167 is a promising novel therapeutic agent for MMBD since OTSSP167 treatment blocked osteoclast activity and stimulated osteoblast activity in vitro, and completely prevented the development of
$\mathrm{MMBD}$ in $\mathrm{MM}$-bearing mice.

Consistent with an osteoclast stimulating activity of factors downstream of MELK, MELK mRNA levels increased during osteoclastogenesis. In addition, we found significant mRNA changes in EZH2 and the negative regulator IRF8 known to influence the crucial differentiation factor NFATC1 which was found to be upregulated during osteo-
A

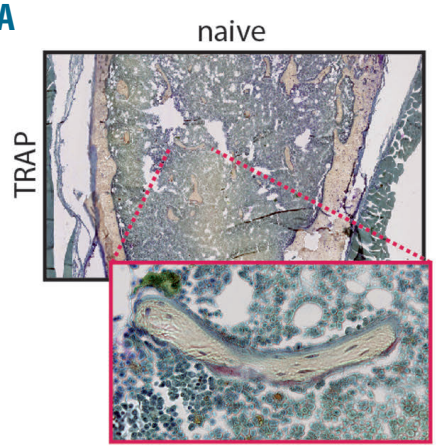

B
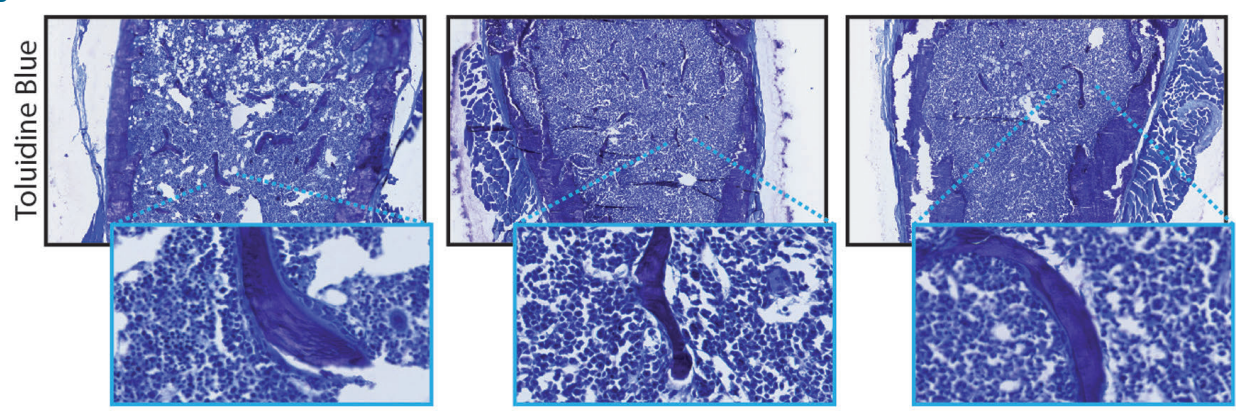

C
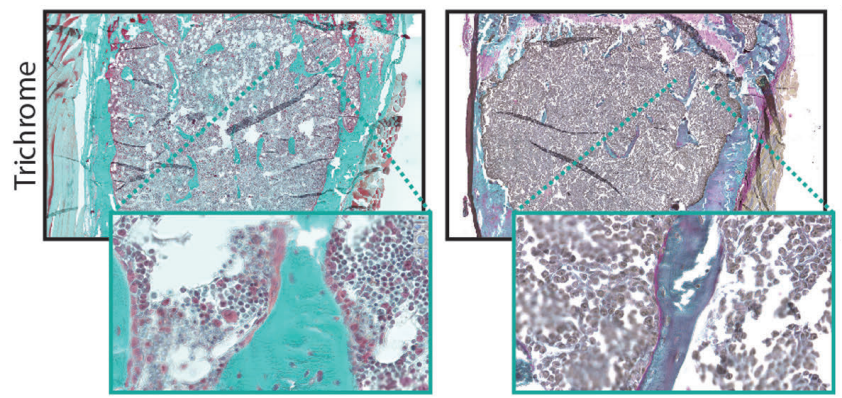

D

E

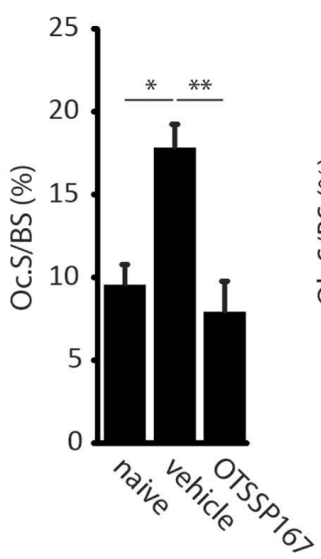

$\mathrm{F}$

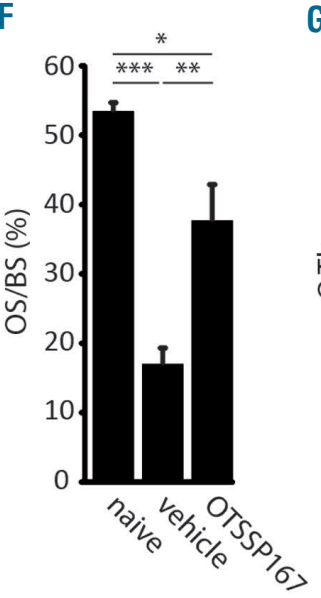

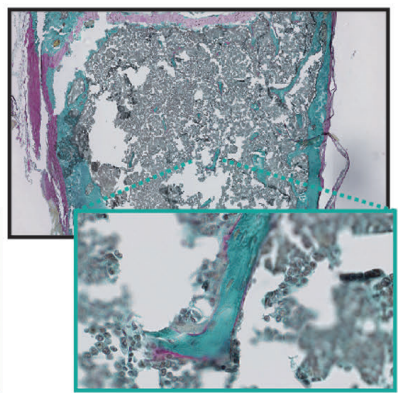

$7.5 \mathrm{mg} / \mathrm{kg} / 2 \mathrm{~d}$ OTSSP 167

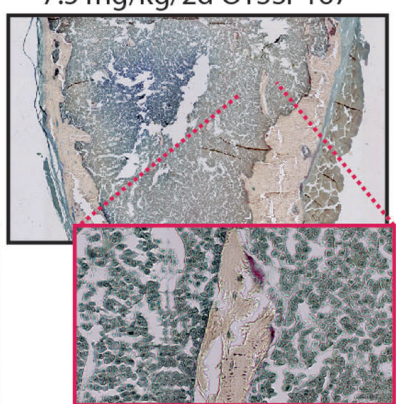

G

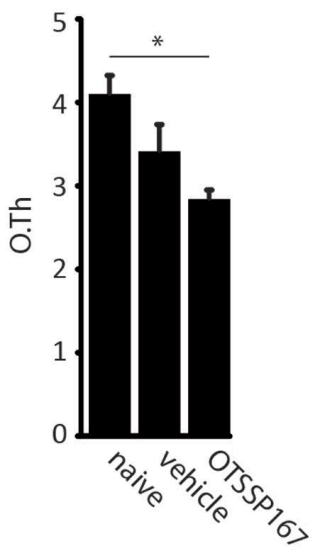

Figure 6. OTSSP167 decreases osteoclast activity and restores osteoblast activity in multiple myeloma-bearing mice. A) Representative images of TRAPstained distal femur sections of naive, vehicle- and OTSSP167treated mice from the 7.5 $\mathrm{mg} / \mathrm{kg} / 2 \mathrm{~d}$ cohort, which showed no effect on MM tumor load. 40X amplifications are shown in the inserts. B) Representative images of toluidine blue-stained distal femur sections from the same cohort. C) Representative images of Masson's trichrome stained distal femur sections from the same cohort. D) Quantification of osteoclast surface (Oc.S/BS). E) Quantification of osteoblast surface (Ob.S/BS). F) Quantification of osteoid surface (OS/BS). G) Quantification of osteoid thickness (O.Th). All data are represented as mean $+/$ - standard error. $*: P<0.05$, $* *: P<0.01, * * *: P<0.001$. 
clast differentiation (illustrated in Online Supplementary Figure S1C). OTSSP167 decreased MELK protein levels in RAW264.7 cells, which agrees with previous studies on various cell types. ${ }^{25,26}$ Of note, OTSSP167 can have off-target effects, which could partially account for the effects of OTSSP167 on osteoclast and osteoblast function. ${ }^{27,28}$ In accordance with previous reports implicating MELK in G2/M transition and proliferation, OTSSP167 decreased the viability of monocytes due to G2/M cell cycle arrest. $^{26,27,29,30}$ The decreased progenitor cell viability resulted in a decreased osteoclast differentiation following OTSSP167 treatment. It is difficult to differentiate between an anti-proliferative effect on progenitor cells and an anti-osteoclast differentiation effect. We observed that 2 pathways, known to interact with MELK, were affected by OTSSP167: i) the transcriptional factor FOXM1 which is implicated in proliferation and ii) the EZH2-IRF8-NFATC1 axis, involved in osteoclast differentiation. Unexpectedly, EZH2 levels increased, which can be explained by the strong link between cell cycle arrest and CDK1/2-dependent EZH2 phosphorylation on different residues (T487), which can either disrupt the binding of EZH2 to other partners of the polycomb repressive complex ${ }^{31}$ or target it for ubiquitin-mediated degradation. $^{32}$ EZH2 thus becomes less functional, which results is a decline in $\mathrm{H} 3 \mathrm{~K} 27$ trimethylation and a de-repression of EZH2 target genes (in our case IRF8). ${ }^{33}$ When mature osteoclasts were treated with OTSSP167, their numbers remained unaltered but matrix resorption was drastically decreased, further corroborating that OTSSP167 has a direct effect on osteoclast activity. Together, our data indicate that OTSSP167 inhibits osteoclast function by hampering monocytic progenitor viability as well as by directly inhibiting mature osteoclast function.

MELK, EZH2 and FOXM1 mRNA levels decreased during osteoblast differentiation, consistent with an inhibitory role of MELK and downstream factors such as EZH2 on osteoblast function. ${ }^{16,17}$ Contrary to osteoclasts and various malignant cells, BMSC-TERT viability was not affected following OTSSP167 treatment at similar concentrations. OTSSP167 treatment increased collagen deposition and strongly stimulated mineralization activity of osteoblasts in vitro and this coincided with an increase in OSX levels but a decrease in RUNX2, OPN and IL-6 mRNA levels. The pro-mineralization activity of OTSSP167 is likely mediated by EZH2 as treatment with an EZH2 inhibitor showed a similar effect. ${ }^{16}$ However, the decrease in RUNX2 expression following OTSSP167 treatment does not correspond with the described role of EZH2 as a suppressor of RUNX2 transcription. ${ }^{16}$ Alternative mechanisms of OTSSP167-induced osteoblast maturation include a marked decrease in the expression of $\mathrm{OPN}$, a non-collagenous bone matrix protein that inhibits matrix mineralization ${ }^{34}$ and IL- $6,{ }^{34}$ a potent growth factor for $\mathrm{MM}$ cells, but also a negative regulator of osteoblast differentiation ${ }^{35}$ and inducer of bone resorption. ${ }^{36}$ Deregulation of these genes in conjunction with the above-mentioned upregulation of $O S X$, a master regulator of mineralization, likely mediates the pro-osteogenic activity of OTSSP167.

The regulation of MELK expression and activity by upstream signaling pathways remains poorly understood including in bone cells. E2F1 $1^{37}$ and FOXM $1^{10}$ have been shown to regulate $M E L K$ gene transcription, the former in osteoblastic MC3T3-E1 cells. Of note, E2F1 has been implicated in increased osteoclastogenesis and osteoblast activity. ${ }^{38,39}$ MELK both regulates and is regulated by one family of MAP kinases, the c-Jun $\mathrm{NH}(2)$-terminal kinases (JNK2), that acts downstream of the RANK-receptor. ${ }^{40}$ Interestingly, JNK2 does not seem to be required for osteoclast differentiation, but rather appears to be involved in osteoclast survival. ${ }^{41}$

Given the promising in vitro data, we assessed whether OTSSP167 would affect the development of MMBD in the murine 5TGM.1 MM model. Although this model reflects human myeloma and associated bone disease in an immunocompetent setting, it should be noted that MM growth in this model progresses rapidly. This cell line allows in vivo studies, however, it also shows a BM-independent growth in vitro and its murine origin may not reflect all the human aspects of myeloma disease (from cytogenetic and molecular point of view). We have previously shown that the dosing schemes we used dosedependently decreased MM tumor load. ' OTSSP167 completely prevented the development of $\mathrm{MMBD}$ at all doses, with no difference between the treatment groups. Both the development of lytic cortical lesions and the large loss of trabecular bone were completely prevented in MMbearing mice treated with OTSSP167. Importantly, this effect also occurred at an OTSSP167 concentration which had no effect on MM tumor load $(7.5 \mathrm{mg} / \mathrm{kg} / 2 \mathrm{~d})$, indicating that OTSSP167 has a direct effect on bone cells and does not solely reduce $\mathrm{MMBD}$ by reducing $\mathrm{MM}$ tumor load. In fact, given the lower concentration of OTSSP167 needed to achieve an anti-MMBD effect, our data suggest that OTSSP167 could exert its anti-MM effect in part by normalizing bone homeostasis. Indeed, the reduced osteoclast numbers following OTSSP167 treatment likely result in reduced myeloma pro-survival factor levels and increased myeloma anti-proliferative factor levels, respectively. Upon treatment with OTSSP167, we found decreased mRNA expression levels of insulin-like growth factor 1 (IGF-1), osteopontin, a proliferation-inducing ligand (APRIL) and interleukin-10 (IL-10) (Online Supplementary Figure S1D).

In conclusion, this study provides a novel approach for the treatment of MMBD. The maintenance of bone anabolic activity by OTSSP167 is promising and warrants further investigation. Reducing MM patient morbidity and mortality via the combined anti-MM and anti-MMBD effect of OTSSP167 holds great clinical promise and our results warrant similar studies in other cancers with bone involvement.

\section{Acknowledgments}

The authors would like to thank the GIGA-imaging platform for their excellent technical assistance.

\section{Funding}

$J M$ and ML are Télévie PhD candidates. The Wilhelminen Cancer Research Institute is supported by the Austrian Forum against Cancer. The laboratory of Hematology was supported by Foundation Against Cancer, the Fonds National de la Recherche Scientifique (F.N.R.S., Belgium) and the Fonds spéciaux de la Recherche (University of Liege). Elodie Duray (research fellow), Erwan Plougonven (post-doctoral researcher) and Frédéric Baron (senior research associate) have a mandate supported by the FNRS. Roy Heusschen is a Télévie postdoctoral research associate. Jo Caers is a post-doctorate clinical specialist funded by the Belgian Foundation against Cancer. 


\section{References}

1. Rollig C, Knop S, Bornhauser M. Multiple myeloma. Lancet. 2015;385(9983):2197. 2208.

2. Roodman GD. Pathogenesis of myeloma bone disease. J Cell Biochem. 2010;109(2):283-291.

3. Heusschen R, Muller J, Duray E, et al. Molecular mechanisms, current management and next generation therapy in myeloma bone disease. Leuk Lymphoma. 2018;59(1):14-28.

4. Galson DL, Silbermann R, Roodman GD. Mechanisms of multiple myeloma bone disease. Bonekey Rep. 2012;1:135.

5. Kennel KA, Drake MT. Adverse effects of bisphosphonates: implications for osteoporosis management. Mayo Clin Proc. 2009;84(7):632-637

6. Silbermann R, Roodman GD. Current controversies in the management of myeloma bone disease. J Cell Physiol. 2016;231(11):2374-2379.

7. Bolomsky A, Heusschen R, Schlangen K, et al. Maternal embryonic leucine zipper kinase is a novel target for proliferationassociated high-risk myeloma. Haematologica. 2018;103(2):325-335.

8. Pickard MR, Green AR, Ellis IO, et al. Dysregulated expression of Fau and MELK is associated with poor prognosis in breast cancer. Breast Cancer Res. 2009;11(4):R60.

9. Du T, Qu Y, Li J, et al. Maternal embryonic leucine zipper kinase enhances gastric cancer progression via the FAK/Paxillin pathway. Mol Cancer. 2014;13:100.

10. Wang Y, Lee YM, Baitsch L, et al. MELK is an oncogenic kinase essential for mitotic progression in basal-like breast cancer cells. Elife. 2014;3:e01763.

11. Ganguly R, Hong CS, Smith LG, Kornblum HI, Nakano I. Maternal embryonic leucine zipper kinase: key kinase for stem cell phenotype in glioma and other cancers. Mol Cancer Ther. 2014;13(6):1393-1398.

12. Joshi K, Banasavadi-Siddegowda Y, Mo X, et al. MELK-dependent FOXM1 phosphorylation is essential for proliferation of glioma stem cells. Stem Cells. 2013;31(6):10511063

13. Kim SH, Joshi K, Ezhilarasan R, et al. EZH2 protects glioma stem cells from radiationinduced cell death in a MELK/FOXM1dependent manner. Stem Cell Reports. 2015;4(2):226-238.

14. Gu C, Yang Y, Sompallae R, et al. FOXM1 is a therapeutic target for high-risk multiple myeloma. Leukemia. 2016;30(4):873-882

15. Fang C, Qiao Y, Mun SH, et al. Cutting edge: EZH2 promotes osteoclastogenesis by epigenetic silencing of the negative regulator IRF8. J Immunol. 2016;196(11):4452-4456.

16. Dudakovic A, Camilleri ET, Xu F, et al. Epigenetic control of skeletal development by the histone methyltransferase Ezh2. J
Biol Chem. 2015;290(46):27604-27617.

17. Dudakovic A, Camilleri ET, Riester SM, et al. Enhancer of zeste homolog 2 inhibition stimulates bone formation and mitigates bone loss caused by ovariectomy in skeletally mature mice. J Biol Chem. 2016; 291(47):24594-24606.

18. Adamik J, Jin S, Sun $\mathrm{O}$, et al. EZH2 or HDAC1 Inhibition reverses multiple myeloma-induced epigenetic suppression of osteoblast differentiation. Mol Cancer Res. 2017;15(4):405-417

19. Heusschen R, Muller J, Binsfeld M, et al. SRC kinase inhibition with saracatinib limits the development of osteolytic bone disease in multiple myeloma. Oncotarget. 2016;7(21):30712-30729.

20. Bolomsky A, Schreder M, Meissner T, et al. Immunomodulatory drugs thalidomide and lenalidomide affect osteoblast differentiation of human bone marrow stromal cells in vitro. Exp Hematol. 2014;42(7):516-525.

21. Binsfeld $M$, Muller J, Lamour $V$, et al. Granulocytic myeloid-derived suppressor cells promote angiogenesis in the context of multiple myeloma. Oncotarget. 2016 7(25):37931-37943.

22. Dempster DW, Compston JE, Drezner MK, et al. Standardized nomenclature, symbols, and units for bone histomorphometry: a 2012 update of the report of the ASBMR Histomorphometry Nomenclature Committee. J Bone Miner Res. 2013;28(1):217

23. Garrett IR, Boyce BF, Oreffo RO, et al. Oxygen-derived free radicals stimulate osteoclastic bone resorption in rodent bone in vitro and in vivo. J Clin Invest. 1990 85(3):632-639.

24. Lee NK, Choi YG, Baik JY, et al. A crucial role for reactive oxygen species in RANKLinduced osteoclast differentiation. Blood. 2005;106(3):852-859.

25. Bolomsky A, Heusschen R, Schlangen K, et al. Maternal embryonic leucine zipper kinase is a novel target for proliferation associated high-risk myeloma. Haematologica. 2018;103(2):325-335

26. Chung S, Suzuki H, Miyamoto $\mathrm{T}$, et al. Development of an orally-administrative MELK-targeting inhibitor that suppresses the growth of various types of human cancer. Oncotarget. 2012;3(12):1629-1640.

27. Simon M, Mesmar F, Helguero L, Williams C. Genome-wide effects of MELK-inhibitor in triple-negative breast cancer cells indicate context-dependent response with p53 as a key determinant. PLoS One. 2017; 12(2):e0172832

28. Lin A, Giuliano CJ, Sayles NM, Sheltzer JM CRISPR/Cas9 mutagenesis invalidates a putative cancer dependency targeted in ongoing clinical trials. Elife. 2017;6.

29. Kohler RS, Kettelhack H, KnipprathMeszaros AM, et al. MELK expression in ovarian cancer correlates with poor out- come and its inhibition by OTSSP167 abrogates proliferation and viability of ovarian cancer cells. Gynecol Oncol. 2017;145(1):159-166.

30. Kato $\mathrm{T}$, Inoue $\mathrm{H}$, Imoto $\mathrm{S}$, et al. Oncogenic roles of TOPK and MELK, and effective growth suppression by small molecular inhibitors in kidney cancer cells. Oncotarget. 2016;7(14):17652-17664

31. Wei Y, Chen Y-H, Li L-Y, et al. CDK1-dependent phosphorylation of EZH2 suppresses methylation of $\mathrm{H} 3 \mathrm{~K} 27$ and promotes osteogenic differentiation of human mesenchymal stem cells. Nature Cell Biology. 2010;13:87

32. Wu SC, Zhang Y. Cyclin-dependent kinase 1 (CDK1)-mediated phosphorylation of enhancer of Zeste 2 (Ezh2) regulates its stability. J Biol Chem. 2011;286(32):2851128519.

33. Lim S, Kaldis P. Cdks, cyclins and CKIs: roles beyond cell cycle regulation. Development. 2013;140(15):3079-3093.

34. Yuan Q, Jiang Y, Zhao X, et al. Increased osteopontin contributes to inhibition of bone mineralization in FGF23-deficient mice. J Bone Miner Res. 2014;29(3):693-704.

35. Kaneshiro S, Ebina K, Shi K, et al. IL-6 negatively regulates osteoblast differentiation through the SHP2/MEK2 and SHP2/Akt2 pathways in vitro. J Bone Miner Metab. 2014;32(4):378-392.

36. Ishimi Y, Miyaura C, Jin CH, et al. IL-6 is produced by osteoblasts and induces bone resorption. J Immunol. 1990;145(10):32973303.

37. Verlinden L, Eelen G, Beullens I, et al. Characterization of the condensin component Cnap1 and protein kinase melk as novel E2F target genes down-regulated by 1,25-dihydroxyvitamin D3. J Biol Chem. 2005;280(45):37319-37330

38. Murata K, Fang C, Terao C, et al. Hypoxiasensitive COMMD1 integrates signaling and cellular metabolism in human macrophages and suppresses osteoclastogenesis. Immunity. 2017;47(1):66-79.e65.

39. Yu S, Yerges-Armstrong LM, Chu Y, Zmuda JM, Zhang Y. E2F1 effects on osteoblast differentiation and mineralization are mediated through up-regulation of frizzled-1. Bone. 2013;56(2):234-241.

40. Caers J, Van Valckenborgh E, Menu E, Van Camp B, Vanderkerken K. Unraveling the biology of multiple myeloma disease: cancer stem cells, acquired intracellular changes and interactions with the surrounding microenvironment. Bull Cancer. 2008;95(3):301313.

41. Amoui M, Sheng MHC, Chen S-T, Baylink DJ, Lau KHW. A transmembrane osteoclastic protein-tyrosine phosphatase regulates osteoclast activity in part by promoting osteoclast survival through c-Src-dependent activation of NF B and JNK2. Arch Biochem Biophys. 2007;463(1):47-59. 